\title{
3D theory of a high-gain free-electron laser based on a transverse gradient undulator
}

\author{
Panagiotis Baxevanis, Yuantao Ding, Zhirong Huang, and Ronald Ruth \\ SLAC National Accelerator Laboratory, Menlo Park, California 94025, USA
}

(Received 21 October 2013; published 6 February 2014)

\begin{abstract}
The performance of a free-electron laser (FEL) depends significantly on the various parameters of the driving electron beam. In particular, a large energy spread in the beam results in a substantial reduction of the FEL gain, an effect which is especially relevant when one considers FELs driven by plasma accelerators or ultimate storage rings. For such cases, one possible solution is to use a transverse gradient undulator (TGU). In this concept, the energy spread problem is mitigated by properly dispersing the electron beam and introducing a linear, transverse field dependence in the undulator. This paper presents a self-consistent theoretical analysis of a TGU-based, high-gain FEL which takes into account three-dimensional (3D) effects, including beam size variations along the undulator. The results of our theory compare favorably with simulation and are used in fast optimization studies of various x-ray FEL configurations.
\end{abstract}

\section{INTRODUCTION}

In recent years, with the successful commissioning of several major facilities around the world [1-4], the freeelectron laser (FEL) has demonstrated its immense value as a tunable source of intense, coherent $\mathrm{x}$ rays. However, achieving the desired quality for the output radiation requires the availability of a high-brightness electron beam to drive the FEL, a fact which places serious restrictions on parameters such as the transverse emittance and the relative energy spread of the e-beam. In this paper, we are predominantly concerned with the latter quantity, which must be much smaller than the gain bandwidth (given by the FEL parameter) if efficient lasing is to occur. This is because a large spread in the energy of the electrons translates into a significant spread in the resonant wavelength (as can be seen from the FEL resonance condition), thus drastically reducing the FEL gain. While standard designs satisfy the aforementioned condition, there exist a number of promising novel concepts for which doing so (in a conventional way) would be very challenging. One prominent example is that of FELs driven by electron beams from laser-plasma accelerators (LPAs) [5]. The latter are characterized by high energy $(\sim 1 \mathrm{GeV})$, low normalized emittance $(\sim 0.1 \mu \mathrm{m})$ and very high peak current (up to $10 \mathrm{kA}$ ), features which make them attractive for compact FEL applications. Unfortunately, they also have a relatively large energy spread $(\sim 1 \%)$ compared to beams from conventional accelerators. Another potential scheme involves the use of the beam from an ultimate storage ring (USR) [6] in a high-gain FEL situated in a bypass close to

Published by the American Physical Society under the terms of the Creative Commons Attribution 3.0 License. Further distribution of this work must maintain attribution to the author(s) and the published article's title, journal citation, and DOI. the ring [7]. This configuration would allow for a high repetition rate but is again limited by the relatively large energy spread $(\sim 0.1 \%)$, as well as the low peak current ( $\sim 100 \mathrm{~A})$. For the case of the LPA-based concepts, some proposals make use of bunch decompression techniques in order to address the energy spread problem [8]. Instead, we will consider an alternative concept, the transverse gradient undulator (TGU), which can also be used in the scheme that involves an ultimate storage ring. The TGU (Fig. 1) is an undulator with canted magnetic poles, so that its vertical field has a linear dependence upon the horizontal position $x$. Using a suitable dispersive element, one can also introduce a linear correlation of the electron energy with $x$. By properly selecting the parameters involved (cant angle and dispersion), one can ensure that electrons with higher than nominal energy are dispersed towards the higher-field region in such a way that the variation in the resonant frequency is minimized.

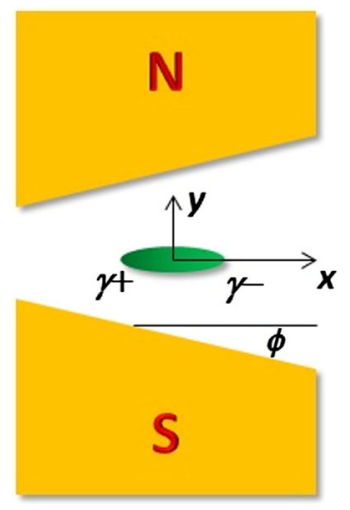

FIG. 1 (color online). Schematic of a transverse gradient undulator (TGU). The undulator poles are canted, which introduces a linear dependence of the vertical field with $x$. The constant field gradient depends on the cant angle $\phi$. 
Originally studied as a way to improve the energy acceptance of low-gain (oscillator) FELs ([9] and [10]), the TGU has recently been considered in the context of its possible application in high-gain devices, particularly those based on LPAs. Specifically, Ref. [11] developed a onedimensional (1D) theoretical model for such a device and investigated the impact of 3D effects through simulation. In this paper, we present a self-consistent, theoretical description of a TGU-based, high-gain FEL in the framework of the Vlasov-Maxwell formalism, including 3D effects such as diffraction, emittance, and betatron oscillations due to the undulator natural focusing. In Sec. II, an outline is given of the single particle equations of motion (the detailed analysis of which is left for the Appendix) and an equation for the radiation amplitude is derived from the VlasovMaxwell equations. When possible, we seek solutions that correspond to the guided eigenmodes of the FEL. In particular, we focus on the fundamental FEL mode and employ a variational technique in order to determine its various parameters. In Sec. III, we present the results of a numerical study which explores two different concepts for a soft $x$ ray, TGU FEL, namely a compact device driven by an LPA and a machine based on an ultimate storage ring. Finally, Sec. IV summarizes our main conclusions.

\section{THEORY}

\section{A. Single particle motion}

To begin with, we assume that the magnetic field of the TGU is given by

$$
\begin{aligned}
& B_{u x}=B_{0} \frac{\alpha}{k_{u}} \sinh \left(k_{u} y\right) \sin \left(k_{u} z\right), \\
& B_{u y}=B_{0}(1+\alpha x) \cosh \left(k_{u} y\right) \sin \left(k_{u} z\right), \\
& B_{u z}=B_{0}(1+\alpha x) \sinh \left(k_{u} y\right) \cos \left(k_{u} z\right),
\end{aligned}
$$

where $k_{u}=2 \pi / \lambda_{u}$ ( $\lambda_{u}$ is the undulator period), $B_{0}$ is the peak on-axis field, and $\alpha$ is the transverse field gradient, which can be related to the cant angle of the undulator poles [11]. This magnetic field satisfies Maxwell's equations in vacuum and reduces to the field of a standard, planar, flatpole undulator for $\alpha \rightarrow 0$. As we have already mentioned, the object of the TGU is to mitigate the negative impact of a large energy spread in the electron beam by significantly reducing the resulting spread in the resonant wavelength $\lambda_{r}$. In order to achieve this, the beam is dispersed in the $x$ direction so that the horizontal position of an electron becomes linearly correlated to its energy $\gamma m c^{2}$ according to $x=\eta \delta$, where $\eta$ is the dispersion, $\delta=\gamma / \gamma_{0}-1$ is the relative energy deviation, and $\gamma_{0} m c^{2}$ is the average electron energy ( $m$ is the electron mass). On the other hand, the introduction of the constant field gradient $\alpha$ leads to a linear $x$ dependence of the undulator parameter $K$, i.e., $K=K_{0}(1+\alpha x)$, where $K_{0}=e B_{0} /\left(m c k_{u}\right)$ is its on-axis value ( $e$ is the electron charge). By selecting the dispersion $\eta$ according to the relation

$$
\eta=\frac{2+K_{0}^{2}}{\alpha K_{0}^{2}}
$$

the resonant condition $\lambda_{r}=\lambda_{u}\left(1+K^{2} / 2\right) /\left(2 \gamma^{2}\right)$ is now satisfied by all the electrons in the beam (up to linear order in $x$ ). Only on-resonance operation of the TGU will be considered in this study, so Eq. (2) is always assumed to be valid.

For a derivation of the single particle equations of motion, we refer to the Appendix. Here, we merely quote the main results. As far as the transverse dynamics is concerned, the TGU is characterized by a horizontal focusing strength $k_{\beta} \sim\left(\eta \gamma_{0}\right)^{-1}$. In this paper, we assume that this focusing effect is weak $\left(k_{\beta} L_{u} \ll 1\right.$, where $L_{u}$ is the undulator length) and exclude it from our treatment. However, we do take into account the vertical natural focusing of the undulator, whose strength $k_{n}$ is given by $k_{n} \approx K_{0} k_{u} /\left(\sqrt{2} \gamma_{0}\right)$. Moreover, we assume that a small, net bending $\left(\sim B_{0} / \gamma_{0}\right)$ due to the asymmetry of the TGU field has been corrected. Thus, the (wiggle-averaged) transverse equations of motion for an electron are $x^{\prime \prime}=0$ and $y^{\prime \prime}=-k_{n}^{2} y$, or, in a more canonical form,

$$
\frac{d x}{d z}=p_{x}, \quad \frac{d p_{x}}{d z}=0, \quad \frac{d y}{d z}=p_{y}, \quad \frac{d p_{y}}{d z}=-k_{n}^{2} y .
$$

In addition, we express the electric field of the radiation (which is linearly polarized along the $x$ direction) as

$$
E_{x}=\frac{1}{2} \int_{0}^{\infty} d \nu E_{\nu}(\mathbf{x}, z) e^{i \nu k_{r}(z-c t)}+\text { c.c. },
$$

where $E_{\nu}(\mathbf{x}, z)$ is the amplitude of the radiation, $\mathbf{x}=(x, y)$ is the transverse position vector, $\nu=\omega / \omega_{r}$ is a scaled frequency variable, $\omega_{r}=c k_{r}=2 \pi c / \lambda_{r}=2 \gamma_{0}^{2} c k_{u} /\left(1+a_{w}^{2}\right)$ is the resonant frequency (with $a_{w}=K_{0} / \sqrt{2}$ ), and c.c. stands for complex conjugate. The pendulum equations for the longitudinal motion are

$$
\frac{d \theta}{d z}=\theta^{\prime}=2 k_{u}\left(\delta-\frac{x}{\eta}\right)-\frac{k_{r}}{2}\left(p_{x}^{2}+p_{y}^{2}+k_{n}^{2} y^{2}\right)
$$

and

$$
\frac{d \delta}{d z}=\kappa_{1} \int_{0}^{\infty} d \nu E_{\nu}(\mathbf{x}, z) e^{-i \Delta \nu k_{u} z} e^{i \nu \theta}+\text { c.c. }
$$

Here, $\theta=k_{u} z+k_{r}(z-c \bar{t})$ is the averaged electron phase $(\bar{t}$ is the electron arrival time averaged over the wiggle motion), $\Delta \nu=\nu-1$ is the frequency detuning parameter, and $\kappa_{1}=e K_{0}[J J] /\left(4 \gamma_{0}^{2} m c^{2}\right)$, where $[J J]=J_{0}\left(Q_{0}\right)-J_{1}\left(Q_{0}\right)$ [with $\left.Q_{0}=K_{0}^{2} /\left(4+2 K_{0}^{2}\right)\right]$ is the well-known factor 
arising from the wiggle averaging. We note the presence of the linear term proportional to $x$ on the right-hand side (RHS) of Eq. (5), which reflects the resonant character of particles with $x=\eta \delta$.

\section{B. Vlasov-Maxwell equations}

The Vlasov-Maxwell formalism allows us to study the interaction between the electron beam and the radiation that is generated during its passage through the TGU in a self-consistent fashion. In this context, the phase-space evolution of the electron beam is described by means of a distribution function $f=f\left(\theta, \delta, x, y, p_{x}, p_{y}, z\right)$. Prior to the onset of saturation, the FEL interaction only adds a small perturbation $\delta f$ to the background distribution $f_{0}$ (i.e., $f=f_{0}+\delta f$, where $|\delta f| \ll f_{0}$ ). Following the standard perturbation analysis [12], it can be shown that the linearized, frequency-domain Vlasov equation for the FEL is (for $\nu>0$ )

$$
\begin{aligned}
\frac{\partial f_{\nu}}{\partial z} & +p_{x} \frac{\partial f_{\nu}}{\partial x}+p_{y} \frac{\partial f_{\nu}}{\partial y}-k_{n}^{2} y \frac{\partial f_{\nu}}{\partial p_{y}}+i \nu \theta^{\prime} f_{\nu} \\
= & -\kappa_{1} \frac{\partial f_{0}}{\partial \delta} E_{\nu} e^{-i \Delta \nu k_{u} z},
\end{aligned}
$$

where $f_{\nu}\left(\delta, x, y, p_{x}, p_{y}, z\right)$ is the Fourier amplitude of the perturbation $\delta f \quad\left[f_{\nu}=(2 \pi)^{-1} \int_{-\infty}^{\infty} d \theta e^{-i \nu \theta} \delta f\right]$. Furthermore, the unperturbed distribution $f_{0}$ is assumed to be $\theta$ independent (which corresponds to a uniform current profile) and satisfies the relation

$$
\frac{\partial f_{0}}{\partial z}+p_{x} \frac{\partial f_{0}}{\partial x}+p_{y} \frac{\partial f_{0}}{\partial y}-k_{n}^{2} y \frac{\partial f_{0}}{\partial p_{y}}=0 .
$$

As far as the normalization of $f_{0}$ is concerned, we adopt the convention of Ref. [13], i.e., $\int_{-\infty}^{\infty} d p_{x} d p_{y} \int_{-\infty}^{\infty} d x d y \times$ $\int_{-\infty}^{\infty} d \delta f_{0}=N_{b} / l_{b}$, where $l_{b}$ and $N_{b}$ are, respectively, the bunch length and the total number of electrons. On the other hand, the evolution of the radiation field along the undulator is governed by the paraxial wave equation, $\left(\frac{\partial}{\partial z}+\frac{\nabla_{\perp}^{2}}{2 i \nu k_{r}}\right) E_{\nu}=-\kappa_{2} e^{i \Delta \nu k_{u} z} \int_{-\infty}^{\infty} d p_{x} d p_{y} \int_{-\infty}^{\infty} d \delta f_{\nu}$,

where $\nabla_{\perp}^{2}=\partial^{2} / \partial \mathbf{x}^{2}=\partial^{2} / \partial x^{2}+\partial^{2} / \partial y^{2}$ is the transverse Laplacian, $\kappa_{2}=e K_{0}[J J] /\left(2 \varepsilon_{0} \gamma_{0}\right)$ and $\varepsilon_{0}$ is the vacuum permittivity. Equations (7)-(9) accurately describe the FEL interaction in the linear regime. Using the method of integration along the unperturbed trajectories (Ref. [12] or [13]), Eq. (7) can be solved in terms of $f_{\nu}$, yielding

$$
\begin{aligned}
f_{\nu}= & -\kappa_{1} \frac{\partial f_{0}}{\partial \delta} \int_{0}^{z} d \zeta E_{\nu}\left(x_{+}, y_{+}, \zeta\right) \\
& \times e^{-i \Delta \nu k_{u} \zeta} \exp \left\{i \nu\left[\theta^{\prime} \xi-\left(k_{u} p_{x} / \eta\right) \xi^{2}\right]\right\}
\end{aligned}
$$

for an initially unmodulated electron beam. In the equation given above, we have defined $\xi=\zeta-z, x_{+}=x+p_{x} \xi$ and $y_{+}=y \cos \left(k_{n} \xi\right)+\left(p_{y} / k_{n}\right) \sin \left(k_{n} \xi\right)$. We then choose a background distribution that corresponds to a dispersed Gaussian beam which is matched to the undulator focusing in the $y$ direction (and thus has a constant vertical size). In this case, $f_{0}$ is given by

$$
\begin{aligned}
f_{0}= & \frac{N_{b} / l_{b}}{(2 \pi)^{5 / 2} \sigma_{x} \sigma_{y} \sigma_{x}^{\prime} \sigma_{y}^{\prime} \sigma_{\delta}} \\
& \times \exp \left[-\frac{\left(x-\eta \delta-p_{x} z_{x}\right)^{2}}{2 \sigma_{x}^{2}}-\frac{p_{x}^{2}}{2 \sigma_{x}^{\prime 2}}\right] \\
& \times \exp \left[-\frac{y^{2}}{2 \sigma_{y}^{2}}-\frac{p_{y}^{2}}{2 \sigma_{y}^{\prime 2}}\right] \exp \left[-\frac{\delta^{2}}{2 \sigma_{\delta}^{2}}\right] .
\end{aligned}
$$

In the above relation, $\sigma_{\delta}$ is the rms energy spread, $z_{x}=$ $z-z_{0}\left(z_{0}\right.$ is a constant offset), $\sigma_{y}$ and $\sigma_{y}^{\prime}$ are the rms vertical beam size and angular divergence while $\sigma_{x}$ and $\sigma_{x}^{\prime}$ are their horizontal counterparts at $z_{x}=0$ (in the absence of dispersion). We also point out that the vertical matching condition is $\sigma_{y}^{\prime} / \sigma_{y}=k_{n}$ and that the beam emittance values are given by $\epsilon_{x}=\sigma_{x} \sigma_{x}^{\prime}$ and $\epsilon_{y}=\sigma_{y} \sigma_{y}^{\prime}$. Inserting Eq. (10) into the RHS of Eq. (9) and performing the $\delta$ integration, we obtain a self-consistent equation for the amplitude of the radiation:

$$
\begin{aligned}
\left(\frac{\partial}{\partial z}+\frac{\nabla_{\perp}^{2}}{2 i k_{r}}\right) E_{\nu}(\mathbf{x}, z)= & -\frac{8 i \rho_{T}^{3} k_{u}^{3}}{2 \pi \sigma_{x}^{\prime} \sigma_{y}^{\prime}} \int_{0}^{z} d \zeta \xi e^{-i \Delta \nu k_{u} \xi} \exp \left[-2\left(\sigma_{\delta}^{\mathrm{ef}}\right)^{2} k_{u}^{2} \xi^{2}\right] \int_{-\infty}^{\infty} d p_{x} d p_{y} E_{\nu}\left(x_{+}, y_{+}, \zeta\right) \\
& \times \exp \left[2 i k_{u} \xi\left[-\frac{\sigma_{x}^{2}}{\sigma_{T}^{2}} \frac{x}{\eta}-\left(\frac{\xi}{2}+\frac{\eta^{2} \sigma_{\delta}^{2}}{\sigma_{T}^{2}} z_{x}\right) \frac{p_{x}}{\eta}\right]\right] \\
& \times \exp \left[-\frac{\left(x-p_{x} z_{x}\right)^{2}}{2 \sigma_{T}^{2}}-\frac{1}{2}\left(\frac{1}{\sigma_{x}^{\prime 2}}+i k_{r} \xi\right) p_{x}^{2}-\frac{1}{2}\left(\frac{1}{\sigma_{y}^{\prime 2}}+i k_{r} \xi\right)\left(p_{y}^{2}+k_{n}^{2} y^{2}\right)\right] .
\end{aligned}
$$

Here, we have approximated $\nu \approx 1$ everywhere except in the detuning term while

$$
\sigma_{T}=\left(\sigma_{x}^{2}+\eta^{2} \sigma_{\delta}^{2}\right)^{1 / 2}=\sigma_{x}\left(1+\frac{\eta^{2} \sigma_{\delta}^{2}}{\sigma_{x}^{2}}\right)^{1 / 2}
$$

is the total horizontal beam size when $\sigma_{x}^{\prime} \rightarrow 0$, 


$$
\rho_{T}=\rho\left(1+\frac{\eta^{2} \sigma_{\delta}^{2}}{\sigma_{x}^{2}}\right)^{-1 / 6}
$$

is the corresponding attenuated FEL parameter [14], and $\sigma_{\delta}^{\text {ef }}$ is a (decreased) effective energy spread given by

$$
\frac{1}{\left(\sigma_{\delta}^{\mathrm{ef}}\right)^{2}}=\frac{1}{\sigma_{\delta}^{2}}+\frac{\eta^{2}}{\sigma_{x}^{2}} \rightarrow \sigma_{\delta}^{\mathrm{ef}}=\sigma_{\delta}\left(1+\frac{\eta^{2} \sigma_{\delta}^{2}}{\sigma_{x}^{2}}\right)^{-1 / 2} .
$$

Optimum operation of the TGU requires a significantly reduced effective energy spread $\left(\eta \sigma_{\delta} / \sigma_{x} \gg 1\right)$, so we can usually approximate $\sigma_{\delta}^{\text {ef }} \approx \sigma_{x} / \eta$. The FEL parameter $\rho$ in absence of dispersion is given by

$$
\rho=\left(\frac{I_{p}}{16 I_{A}} \frac{K_{0}^{2}[J J]^{2}}{\gamma_{0}^{3} \sigma_{x} \sigma_{y} k_{u}^{2}}\right)^{1 / 3},
$$

where $I_{p}=e c N_{b} / l_{b}$ is the peak current and $I_{A}=$ $4 \pi \varepsilon_{0} m c^{3} / e \approx 17 \mathrm{kA}$ is the Alfven current. We note that, for a conventional FEL, the energy spread requirement would be $\sigma_{\delta} \ll \rho$. Equation (12) incorporates all the threedimensional effects under consideration, including the variation of the horizontal beam size with $z$. The latter is a consequence of the $z$ dependence of $f_{0}$ when $\epsilon_{x} \neq 0$, which also causes the integral kernel in Eq. (12) to depend explicitly upon $z$ (through $z_{x}$ ).

\section{Eigenmode formalism}

To simplify our treatment, we consider the case of vanishing horizontal emittance (i.e., the case with $\sigma_{x}^{\prime} \rightarrow 0$ but $\sigma_{y}^{\prime} \neq 0$ ), when both sizes of the electron beam are constant. This regime is relevant for a TGU FEL driven by an ultimate storage ring and also allows for a standardized description in terms of the self-similar, guided eigenmodes of the FEL. The latter are solutions of the form $E_{\nu}(\mathbf{x}, z)=A(\mathbf{x}) e^{i \mu z}$, where $\mu$ is the (constant) complex growth rate and $A(\mathbf{x})$ is the mode profile. Using Eq. (12), we can show that the eigenmode equation satisfied by the mode profile and the growth rate is

$$
\begin{aligned}
\left(\mu-\frac{\nabla_{\perp}^{2}}{2 k_{r}}\right) A(\mathbf{x})= & -\frac{8 \rho_{T}^{3} k_{u}^{3}}{\sqrt{2 \pi} \sigma_{y}^{\prime}} \exp \left(-\frac{x^{2}}{2 \sigma_{T}^{2}}-\frac{y^{2}}{2 \sigma_{y}^{2}}\right) \int_{-\infty}^{0} d \xi \xi e^{i\left(\mu-\Delta \nu k_{u}\right) \xi} e^{-2\left(\sigma_{\delta}^{\mathrm{ef}}\right)^{2} k_{u}^{2} \xi^{2}} \exp \left[-2 i \frac{\sigma_{x}^{2}}{\sigma_{T}^{2}} \frac{x}{\eta} k_{u} \xi\right] \\
& \times \int_{-\infty}^{\infty} d p_{y} A\left(x, y_{+}\right) \exp \left[-\frac{p_{y}^{2}}{2 \sigma_{y}^{\prime 2}}-\frac{i k_{r} \xi}{2}\left(p_{y}^{2}+k_{n}^{2} y^{2}\right)\right],
\end{aligned}
$$

where only growing modes (with $\operatorname{Im}[\mu]<0$ ) have been considered. We make use of a variational technique in order to obtain an approximate solution for the Gaussian-like, fundamental mode [15], which is typically the fastest-growing of the FEL eigenmodes. We begin by assuming a trial solution of the form $A(\mathbf{x}) \propto \exp \left(-a_{x} x^{2}+b x\right) \exp \left(-a_{y} y^{2}\right)$ for the transverse profile, where the $b x$ term has been added to account for the asymmetry (under the reflection $x \rightarrow-x$ ) introduced by the exponent that is proportional to $x / \eta$ in Eq. (17). We then multiply both sides of the eigenmode equation by $A(\mathbf{x})$ and integrate over the transverse position. The result of this manipulation is the relation

$$
\begin{aligned}
F\left(a_{x}, a_{y}, b, \mu\right) \equiv & \mu+\frac{a_{x}+a_{y}}{2 k_{r}}+\frac{8 \rho_{T}^{3} k_{u}^{3} \sqrt{a_{x}} \sqrt{a_{y}}}{\sqrt{a_{x}+1 /\left(4 \sigma_{T}^{2}\right)}} \int_{-\infty}^{0} d \xi \xi \exp \left(\frac{\left(b-\frac{1}{\eta}\left(\sigma_{x}^{2} / \sigma_{T}^{2}\right) i k_{u} \xi\right)^{2}}{2 a_{x}+1 /\left(2 \sigma_{T}^{2}\right)}-\frac{b^{2}}{2 a_{x}}\right) \\
& \times \exp \left[i\left(\mu-\Delta \nu k_{u}\right) \xi-2\left(\sigma_{\delta}^{\mathrm{ef}}\right)^{2} k_{u}^{2} \xi^{2}\right] /\left[a_{y}^{2} \sigma_{y}^{2} \sin ^{2}\left(k_{n} \xi\right)\right. \\
& \left.+a_{y}\left(1+i k_{r} \sigma_{y}^{2} \xi\right)+\left(1 /\left(4 \sigma_{y}^{2}\right)\right)\left(1+i k_{r} \sigma_{y}^{\prime 2} \xi\right)^{2}\right]^{1 / 2}=0,
\end{aligned}
$$

which expresses $\mu$ in terms of $a_{x}, a_{y}$, and $b$. The next step is to use the variational conditions, namely $\partial \mu / \partial a_{x}=0$, $\partial \mu / \partial a_{y}=0$, and $\partial \mu / \partial b=0$, which stem from the fact that a first order variation of the mode profile yields only a second order variation in the growth rate. In particular, we differentiate Eq. (18) with respect to $a_{x}$, which yields $\partial F / \partial a_{x}+$ $(\partial F / \partial \mu)\left(\partial \mu / \partial a_{x}\right)=0$ or-in view of the variational condition- $\partial F / \partial a_{x}=0$. In a similar way, we also have the relations $\partial F / \partial a_{y}=0$ and $\partial F / \partial b=0$. Solving the above-mentioned derivative relations in conjunction with Eq. (18), we obtain an approximation to the growth rate and the parameters of the fundamental mode. When emittance and focusing effects in both transverse directions are negligible, as is usually the case with LPA-based examples, we can employ a parallel-beam model for both $x$ and $y$, in which case Eqs. (17) and (18) reduce to

$$
\begin{aligned}
\left(\mu-\frac{\nabla_{\perp}^{2}}{2 k_{r}}\right) A(\mathbf{x})= & -8 \rho_{T}^{3} k_{u}^{3} A(\mathbf{x}) \exp \left(-\frac{x^{2}}{2 \sigma_{T}^{2}}-\frac{y^{2}}{2 \sigma_{y}^{2}}\right) \\
& \times \int_{-\infty}^{0} d \xi \xi e^{i\left(\mu-\Delta \nu k_{u}\right) \xi} e^{-2\left(\sigma_{\delta}^{\mathrm{ef}}\right)^{2} k_{u}^{2} \xi^{2}} \\
& \times \exp \left[-2 i \frac{\sigma_{x}^{2} x}{\sigma_{T}^{2}} \frac{x}{\eta} k_{u} \xi\right]
\end{aligned}
$$


and

$$
\begin{aligned}
F\left(a_{x}, a_{y}, b, \mu\right) \equiv & \mu+\frac{a_{x}+a_{y}}{2 k_{r}}+\frac{8 \rho_{T}^{3} k_{u}^{3} \sqrt{a_{x}} \sqrt{a_{y}}}{\sqrt{a_{x}+1 /\left(4 \sigma_{T}^{2}\right)} \sqrt{a_{y}+1 /\left(4 \sigma_{y}^{2}\right)}} \int_{-\infty}^{0} d \xi \xi \exp \left[i\left(\mu-\Delta \nu k_{u}\right) \xi-2\left(\sigma_{\delta}^{\mathrm{ef}}\right)^{2} k_{u}^{2} \xi^{2}\right] \\
& \times \exp \left(\frac{\left(b-\frac{1}{\eta}\left(\sigma_{x}^{2} / \sigma_{T}^{2}\right) i k_{u} \xi\right)^{2}}{2 a_{x}+1 /\left(2 \sigma_{T}^{2}\right)}-\frac{b^{2}}{2 a_{x}}\right)=0
\end{aligned}
$$

respectively. The integral on the RHS of the above relation can be expressed in terms of error functions, allowing for faster numerical calculations. In particular, we find

$$
\begin{aligned}
F\left(a_{x}, a_{y}, b, \mu\right) \equiv & \mu+\frac{a_{x}+a_{y}}{2 k_{r}}+\frac{8 \rho_{T}^{3} k_{u}^{3} \sqrt{a_{x}} \sqrt{a_{y}}}{\sqrt{a_{x}+1 /\left(4 \sigma_{T}^{2}\right)} \sqrt{a_{y}+1 /\left(4 \sigma_{y}^{2}\right)}} \\
& \times \mathrm{e}^{A_{0}}\left[\frac{\sqrt{\pi} A_{1}}{4 A_{2}^{3 / 2}} \exp \left(\frac{A_{1}^{2}}{4 A_{2}}\right) \operatorname{erfc}\left(\frac{A_{1}}{2 A_{2}^{1 / 2}}\right)-\frac{1}{2 A_{2}}\right]=0,
\end{aligned}
$$

where $\operatorname{erfc}(u)=1-(2 / \sqrt{\pi}) \int_{0}^{u} e^{-s^{2}} d s$ is the complementary error function and

$$
A_{0}=-\frac{b^{2}}{4 a_{x}\left(2 a_{x} \sigma_{T}^{2}+1 / 2\right)}, \quad A_{1}=i\left[\mu-\Delta \nu k_{u}-2 \frac{\sigma_{x}^{2}}{\sigma_{T}^{2}} \frac{k_{u} b / \eta}{2 a_{x}+1 /\left(2 \sigma_{T}^{2}\right)}\right], \quad A_{2}=\frac{\sigma_{x}^{4}}{\sigma_{T}^{4}} \frac{\left(k_{u} / \eta\right)^{2}}{2 a_{x}+1 /\left(2 \sigma_{T}^{2}\right)}+2\left(\sigma_{\delta}^{\mathrm{ef}}\right)^{2} k_{u}^{2}
$$

Once the growth rate and the mode parameters are known, one can calculate the power gain length $L_{g}=-1 /(2 \operatorname{Im}[\mu])$ as well as the mode sizes $\sigma_{r x}=\left(4 \operatorname{Re}\left[a_{x}\right]\right)^{-1 / 2}$ and $\sigma_{r y}=$ $\left(4 \operatorname{Re}\left[a_{y}\right]\right)^{-1 / 2}$ (we clarify that Re and Im refer, respectively, to real and imaginary part). The latter two quantities are defined by the relations $\sigma_{r x}=\sqrt{\left\langle x^{2}\right\rangle-x_{c}^{2}}$ and $\sigma_{r y}=\sqrt{\left\langle y^{2}\right\rangle}$, where the average uses the mode intensity profile $|A(\mathbf{x})|^{2}$ as a weighting function (i.e., we have $\left\langle x^{2}\right\rangle=\int_{-\infty}^{\infty} d^{2} \mathbf{x}|A(\mathbf{x})|^{2} x^{2} / \int_{-\infty}^{\infty} d^{2} \mathbf{x}|A(\mathbf{x})|^{2}$ etc. $)$ and $x_{c}=$ $\operatorname{Re}[b] /\left(2 \operatorname{Re}\left[a_{x}\right]\right)$ is the horizontal centroid of the mode. In the $1 \mathrm{D}$ limit, both electron beam sizes are much larger than the diffraction size $\sigma_{D} \sim\left(\rho_{T} k_{r} k_{u}\right)^{-1 / 2}$, that is $\sigma_{T}, \sigma_{y} \gg \sigma_{D}$. As a result, we have $\sigma_{r x} \sim \sqrt{\sigma_{T} \sigma_{D}} \ll \sigma_{T}, \sigma_{r y} \sim \sqrt{\sigma_{y} \sigma_{D}} \ll$ $\sigma_{y}$ and also $x_{c} \ll \sigma_{T}$. In view of the above properties, the results of the parallel-beam analysis are considerably simplified. In particular, we can approximate $a_{x}+$ $1 /\left(4 \sigma_{T}^{2}\right) \approx a_{x}$ and $a_{y}+1 /\left(4 \sigma_{y}^{2}\right) \approx a_{y}$ while the term proportional to $a_{x}+a_{y}$ in the first line of Eq. (21) is of order $\sigma_{D} / \sigma_{T}+\sigma_{D} / \sigma_{y}$ when compared to $\mu$ and can be dropped. Using similar arguments, we can show that all the terms in Eq. (22) that contain the mode parameters are negligible. Thus, Eq. (21) becomes

$$
\mu+\frac{8 \rho_{T}^{3} k_{u}^{3}}{2 A_{2}}\left[\frac{\sqrt{\pi} A_{1}}{2 A_{2}^{1 / 2}} \exp \left(\frac{A_{1}^{2}}{4 A_{2}}\right) \operatorname{erfc}\left(\frac{A_{1}}{2 A_{2}^{1 / 2}}\right)-1\right]=0
$$

where $A_{1}=i\left(\mu-\Delta \nu k_{u}\right)$ and $A_{2}=2\left(\sigma_{\delta}^{\mathrm{ef}}\right)^{2} k_{u}^{2}$. In the limit of vanishing effective energy spread $\left(\sigma_{\delta}^{\mathrm{ef}} \rightarrow 0\right)$, the error function can be expressed by the asymptotic formula $\operatorname{erfc}(u)=\left[e^{-u^{2}} /(\sqrt{\pi} u)\right]\left[1-1 /\left(2 u^{2}\right)+\cdots\right] \quad$ (valid for $|u| \gg 1)$, in which case we recover the well-known cubic dispersion relation $\mu-8 \rho_{T}^{3} k_{u}^{3} / A_{1}^{2}=\mu+8 \rho_{T}^{3} k_{u}^{3} /$ $\left(\mu-\Delta \nu k_{u}\right)^{2}=0$. For zero detuning, we obtain the equation $\mu^{3}=-8 \rho_{T}^{3} k_{u}^{3}$, whose growing solution is $\mu=(1-i \sqrt{3}) \rho_{T} k_{u}$.

\section{NUMERICAL RESULTS}

To illustrate our theoretical analysis, we have considered two FEL parameter sets, both of which refer to TGU-based

TABLE I. Undulator and electron beam parameters.

\begin{tabular}{lcc}
\hline \hline Parameter & LPA & USR \\
\hline Undulator parameter $K_{0}$ & 2 & 3.68 \\
Undulator period $\lambda_{u}$ & $1 \mathrm{~cm}$ & $2 \mathrm{~cm}$ \\
Beam energy $\gamma_{0} m c^{2}$ & $1 \mathrm{GeV}$ & $4.5 \mathrm{GeV}$ \\
Resonant wavelength $\lambda_{r}$ & $3.9 \mathrm{~nm}$ & $1 \mathrm{~nm}$ \\
Peak current $I_{p}$ & $10 \mathrm{kA}$ & $200 \mathrm{~A}$ \\
Energy spread $\sigma_{\delta}$ & $10^{-2}$ & $1.5 \times 10^{-3}$ \\
Normalized emittance $\gamma_{0} \epsilon_{x}$ & $0.1 \mu \mathrm{m}$ & $0.0123 \mu \mathrm{m}$ \\
Normalized emittance $\gamma_{0} \epsilon_{y}$ & $0.1 \mu \mathrm{m}$ & $1.23 \mu \mathrm{m}$ \\
Horizontal size $\sigma_{x}$ & $11.3 \mu \mathrm{m}$ & $8.3 \mu \mathrm{m}$ \\
Vertical size $\sigma_{y}$ & $11.3 \mu \mathrm{m}$ & $38.7 \mu \mathrm{m}$ \\
FEL parameter $\rho$ & $6 \times 10^{-3}$ & $6 \times 10^{-4}$ \\
\hline \hline
\end{tabular}




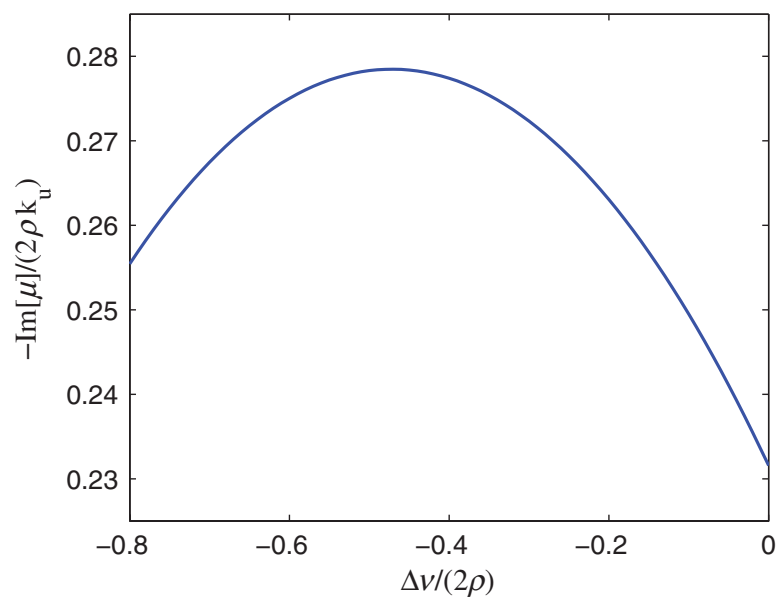

FIG. 2 (color online). Negative imaginary part of the fundamental FEL growth rate $\mu$ (in units of $2 \rho k_{u}$ ) as a function of the detuning $\Delta \nu$ (in units of $2 \rho$ ) for $\eta=3.5 \mathrm{~mm}$ (LPA set).

concepts for soft x-ray machines (Table I). The first set describes an FEL driven by a laser-plasma accelerator [11] while the second set corresponds to a machine that utilizes the electron beam from the proposed PEP-X ultimate storage ring [7]. Starting with the LPA parameter set, we initially select a dispersion $\eta=3.5 \mathrm{~mm}$, which yields (roughly) a $35 \mu \mathrm{m} \times 10 \mu \mathrm{m}$ electron beam. Using the parallel-beam model-Eq. (20) or (21)—we study the variation of the main properties of the fundamental mode with respect to the detuning parameter. In particular, in Fig. 2, we plot the negative imaginary part of the scaled, fundamental growth rate $\hat{\mu}=\mu /\left(2 \rho k_{u}\right)$ as a function of the scaled detuning $\hat{\nu}=\Delta \nu /(2 \rho)$ while Fig. 3 shows the frequency dependence of the scaled mode sizes. For $\hat{\nu} \approx$ -0.5 , the growth rate has a maximum value of $-\operatorname{Im}[\hat{\mu}]_{\max } \approx$ 0.28 . This corresponds to a frequency-optimized gain length

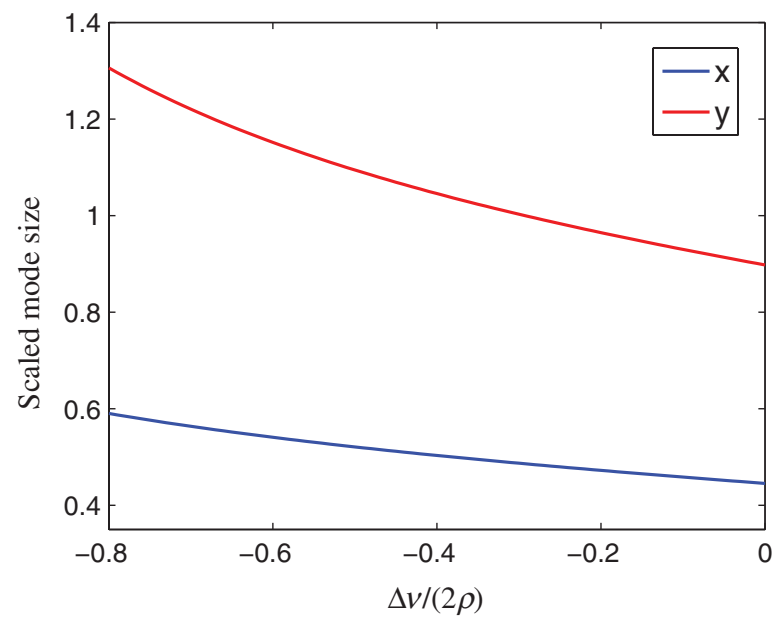

FIG. 3 (color online). Scaled mode sizes as a function of the scaled detuning. In particular, the blue curve shows the variation of $\sigma_{r x} / \sigma_{T}$ while the red one corresponds to $\sigma_{r y} / \sigma_{y}(\eta=3.5 \mathrm{~mm}$, LPA set). of $L_{q}=\sqrt{3} L_{0} /(2|\operatorname{Im}[\hat{\mu}]|) \approx 22.3 \mathrm{~cm}$, where $L_{0}=\lambda_{u} /$ $(4 \pi \sqrt{3} \rho) \approx 7.3 \mathrm{~cm}$ is the $1 \mathrm{D}$ gain length. Moreover, we note that the mode size in both $x$ and $y$ is comparable to the electron beam size and increases as one moves towards longer wavelength (negative detuning). Our next step is to investigate whether or not the dispersion value we started with is an optimum one. To this end, we first point out that the analytical formula of Eq. (21) facilitates the fast numerical calculation of the frequency-optimized gain length as a function of the dispersion. The results of such an analysis are shown in Fig. 4. For comparison, we have also included optimized gain length values derived from the 1D fitting formula [11],

$$
L_{g} \approx \frac{\lambda_{u}}{4 \pi \sqrt{3} \rho_{T}}\left[1+\frac{\left(\sigma_{\delta}^{\mathrm{ef}}\right)^{2}}{\rho_{T}^{2}}\right],
$$

where we also use the approximation $\sigma_{\delta}^{\text {ef }} \approx \sigma_{x} / \eta$. As expected, the gain length estimates from the 3D theory are larger than those given by the 1D formalism. However, the functional behavior is the same in both cases, in that the optimized gain length attains a minimum for a particular dispersion ( 5 or $7 \mathrm{~mm}$ in our case). For dispersion values smaller than the optimum (where the effective energy spread is still large), the gain length curve is considerably steeper than for large $\eta$, where the large horizontal beam size eventually dominates the variation of $L_{g}$. Lastly, we note that for $\eta=1 \mathrm{~cm}$ (a value close to the optimum), we obtain an optimized gain length of slightly more than $20 \mathrm{~cm}$. This is in agreement with the SASE simulations presented in [11], which showed saturation within $5 \mathrm{~m}$ of the undulator for this particular dispersion.

Next, we turn to the parameter set describing a TGU FEL based on the PEP-X ultimate storage ring. In this case, we first need to point out that the undulator structure is rotated

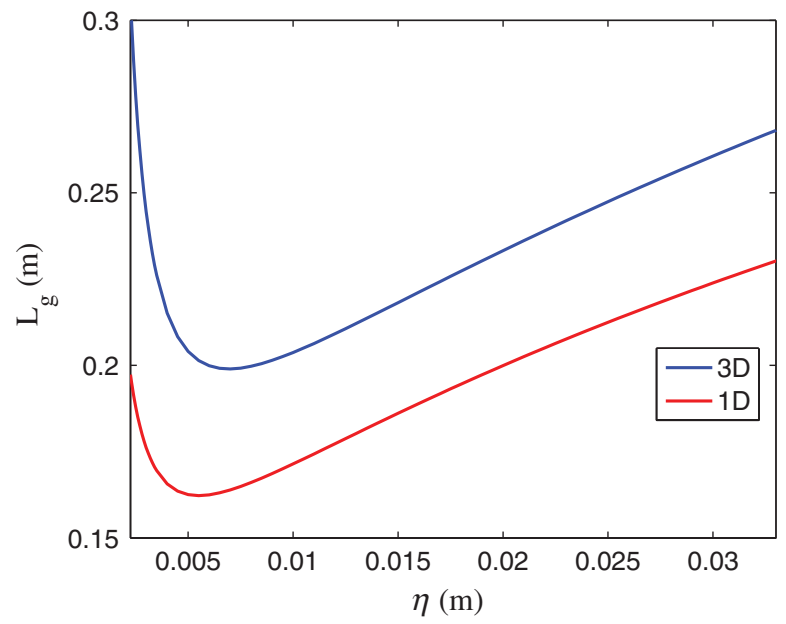

FIG. 4 (color online). Frequency-optimized gain length as a function of dispersion for the LPA parameters. The data shown were derived using the parallel-beam theory (blue) and the 1D formula of Eq. (24) (red). 
by $90^{\circ}$, so that its $x$ direction (i.e., the one in which the dispersion and the field gradient are introduced) becomes perpendicular to the horizontal plane of the ring. This is done in order to take advantage of the much smaller equilibrium emittance in the vertical plane of the ring (for our parameters, the emittance ratio $\epsilon_{x} / \epsilon_{y}$ is $1: 100$ ). To avoid confusion, we emphasize that the terms "horizontal" $(x)$ and "vertical" $(y)$ direction always refer, respectively, to the dispersive direction and the direction perpendicular to it. Moreover, we remind the reader that the configuration under study involves a single-pass, high-gain FEL that is located in a bypass line next to the ring. Therefore, we expect any potential restrictions on the achievable undulator strength due to horizontal aperture/gap requirements to be less important here than in the case of standard storage ring operation. After clarifying these preliminary points, we proceed to the main part of our analysis. For an initial dispersion $\eta=3.5 \mathrm{~cm}$, we perform a steady-state GENESIS simulation [16] assuming a Gaussian input field. Since our objective is to verify the results of that part of our theory which takes into account vertical emittance and undulator focusing, we assume that the electron beam is matched to the undulator in the $y$ direction (with a natural beta function $\beta_{n}=1 / k_{n} \approx 12 \mathrm{~m}$ ) but neglect its horizontal emittance. The results of the simulation are shown in Figs. 5 and 6, where we plot the local power growth rate and the radiation size as functions of $z$. Also included are the variational values for the inverse gain length and the size of the fundamental FEL mode. We stress that, since GENESIS defines the radiation size $\sigma_{r}$ as $\sqrt{\left\langle x^{2}+y^{2}\right\rangle}$, the corresponding value for the mode size is $\sqrt{\sigma_{r x}^{2}+\sigma_{r y}^{2}+x_{c}^{2}}$. Good agreement is observed between simulation and

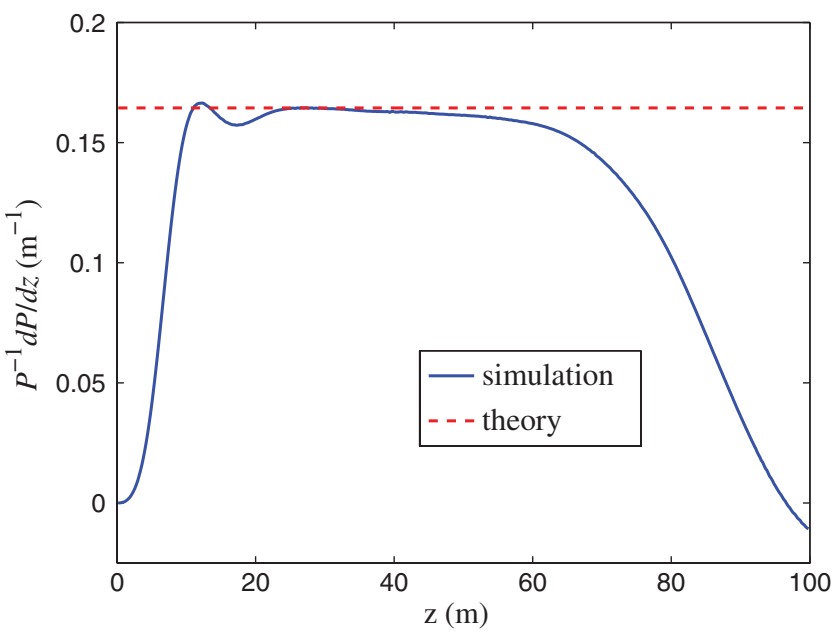

FIG. 5 (color online). Local power growth rate $P^{-1} d P / d z$ (where $P$ is the radiation power) as obtained from a steady-state GENESIS simulation (solid line-USR set $/ \eta=3.5 \mathrm{~cm}$ ). The dashed line corresponds to the inverse power gain length $1 / L_{g}$ of the fundamental mode, calculated using the variational theory.

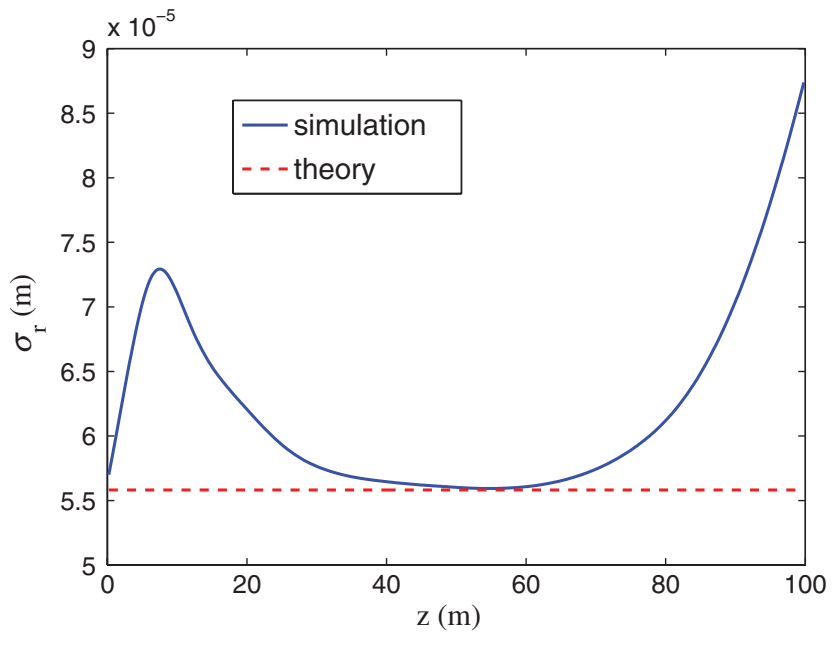

FIG. 6 (color online). Radiation size as obtained from GENESIS (solid line-USR set $/ \eta=3.5 \mathrm{~cm}$ ). Also included is the variational estimate of the fundamental mode size (dashed line).

theory in the high-gain part of the linear regime, where the fundamental mode is expected to be dominant.

Having checked the theoretical formalism, we use it to estimate the optimum value of the dispersion. In Fig. 7, we plot the power gain length in terms of the dispersion for different values of the detuning parameter, using both the simplified parallel-beam theory and the more accurate model described by Eqs. (17) and (18). As expected, the FEL growth rate is suppressed by the included vertical emittance effect and the gain length curves are shifted upwards. From the dashed curves, we obtain an optimum gain length of slightly less than $6 \mathrm{~m}$ for a dispersion $\eta \approx 5 \mathrm{~cm}$. Finally, it is worth noting that for such dispersion

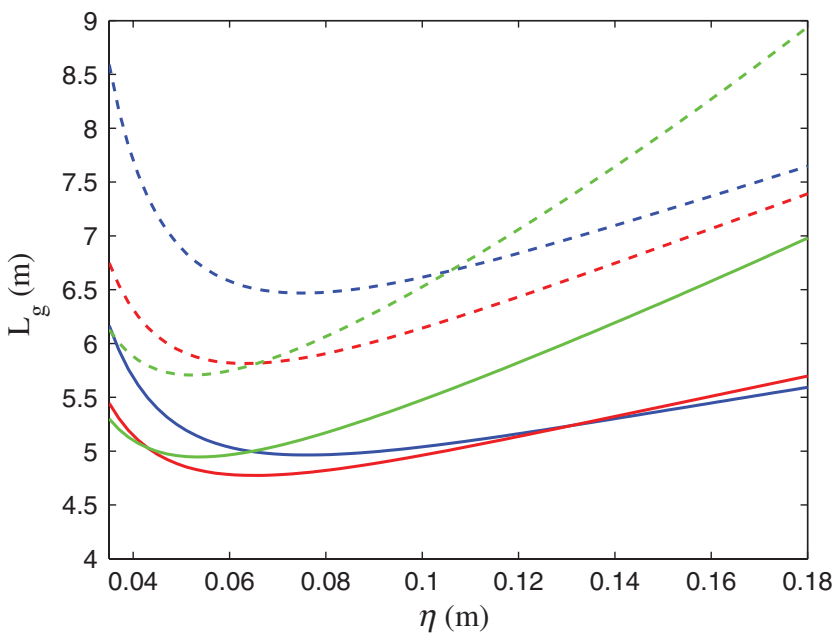

FIG. 7 (color online). Gain length as a function of dispersion for $\Delta \nu /(2 \rho)=0.0 /-0.2 /-0.4$ (blue/red/green-USR set). Included are data from a parallel beam analysis (solid lines) and the model which includes vertical emittance and focusing (dashed lines). 
values (i.e., a few $\mathrm{cm}$ ), the electron beam (unlike the LPA case) is approximately round with sizes $\sim 40-50 \mu \mathrm{m}$. This results in the additional advantage of increased transverse coherence for the output radiation [7], as the transverse profile of the fundamental FEL mode is also approximately symmetric and roughly matches that of the electron beam.

\section{CONCLUSIONS}

We have developed a theoretical framework for the study of a TGU-based, high-gain FEL which takes into account three-dimensional effects such as emittance and undulator focusing (the latter in the nondispersive plane only), as well as electron beam size variations with $z$. Starting from the Vlasov-Maxwell equations for the FEL, we derived a self-consistent equation which governs the evolution of the radiation field in the linear regime. For the special case of constant electron beam sizes, we use this equation to formulate a more standard description of the FEL in terms of its eigenmodes. In particular, we focus on the fundamental FEL mode, the parameters of which are obtained using a variational approach. For sufficiently small emittance in both transverse planes, we can use a parallelbeam model which yields fully analytical expressions and facilitates the fast calculation of the mode properties. The results of our analysis have been used in numerical studies of two soft x-ray TGU FEL configurations, involving novel concepts based on laser-plasma accelerators and ultimate storage rings. Good agreement with simulation has been demonstrated while our theory extends already existing 1D results. We believe that this formalism will be useful in further understanding the physics of a TGU FEL and in providing an analytical way for optimizing its parameters.

\section{ACKNOWLEDGMENTS}

We would like to thank R. Lindberg for useful discussions.

\section{APPENDIX: EQUATIONS OF MOTION}

This section contains a derivation of the equations of motion for a single electron in the combined field of the radiation and the undulator. In general, it is also necessary to superimpose an additional, corrector magnetostatic field of the form

$$
\begin{aligned}
& B_{c x}=\left(\partial B_{c y} / \partial x\right) y=B_{0 c} \alpha_{c} y, \\
& B_{c y}=B_{0 c}\left(1+\alpha_{c} x\right), \\
& B_{c z}=0
\end{aligned}
$$

in order to keep the dispersion function constant along the undulator (we shall verify this assertion later on). We begin by considering the motion in the transverse plane. Disregarding the direct influence of the radiation field, the horizontal motion is governed by the equation

$$
\frac{d\left(\gamma m v_{x}\right)}{d t}=v_{z} \frac{d\left(\gamma m v_{z} x^{\prime}\right)}{d z}=-e\left(v_{y} B_{z}-v_{z} B_{y}\right),
$$

where $v_{x, y, z}$ are the velocity components, $B_{y}=B_{u y}+B_{c y}$, $B_{z}=B_{u z}$ and the prime denotes differentiation with respect to $z$. Approximating $v_{z} \approx c$ (which also implies that $v_{y} \approx c y^{\prime}$ ) and neglecting the variation of $\gamma$ with $z$, we obtain

$$
\begin{aligned}
x^{\prime \prime} \approx & \frac{e\left[B_{y}-y^{\prime} B_{z}\right]}{\gamma m c} \\
= & \frac{e}{\gamma m c}\left[B(x) \cosh \left(k_{u} y\right) \sin \left(k_{u} z\right)\right. \\
& \left.+B_{c}(x)-y^{\prime} B(x) \sinh \left(k_{u} y\right) \cos \left(k_{u} z\right)\right],
\end{aligned}
$$

where we have used Eq. (1) and introduced the abbreviations $B(x)=B_{0}(1+\alpha x)$ and $B_{c}(x)=B_{0 c}\left(1+\alpha_{c} x\right)$. In a similar way, we find that the equation of motion in the vertical direction is

$$
\begin{aligned}
y^{\prime \prime} \approx & \frac{e\left[B_{z} x^{\prime}-B_{x}\right]}{\gamma m c} \\
= & \frac{e}{\gamma m c}\left[x^{\prime} B(x) \sinh \left(k_{u} y\right) \cos \left(k_{u} z\right)\right. \\
& \left.-B_{0}\left(\alpha / k_{u}\right) \sinh \left(k_{u} y\right) \sin \left(k_{u} z\right)-B_{0 c} \alpha_{c} y\right],
\end{aligned}
$$

where $B_{x}=B_{u x}+B_{c x}$. Since we are dealing with a planar undulator, the horizontal motion of the electron can be decomposed into a fast, small-amplitude wiggle motion $x_{w}$ and a much slower, large-amplitude "averaged" motion $\bar{x}$, i.e., $x=\bar{x}+x_{w}$. As we shall see, a similar conclusion actually holds for the $y$ direction as well, so that we can write $y=\bar{y}+y_{w}$. A relation for $x_{w}$ can be derived by replacing the RHS of Eq. (A3) with its leading oscillatory term. In particular, we obtain the equation

$$
x_{w}^{\prime \prime} \approx \frac{e B(\bar{x})}{\gamma m c} \cosh \left(k_{u} \bar{y}\right) \sin \left(k_{u} z\right),
$$

which can be solved approximately $([10],[12])$ to give

$$
x_{w}^{\prime} \approx-\frac{e B(\bar{x})}{\gamma m c k_{u}} \cosh \left(k_{u} \bar{y}\right) \cos \left(k_{u} z\right)
$$

and

$$
x_{w} \approx-\frac{e B(\bar{x})}{\gamma m c k_{u}^{2}} \cosh \left(k_{u} \bar{y}\right) \sin \left(k_{u} z\right) .
$$

The vertical, fast oscillatory motion $y_{w}$ is induced by the $x$ component of the undulator field and is governed by

$$
y_{w}^{\prime} \approx \frac{K_{0}}{\gamma} \frac{\alpha}{k_{u}} \sinh \left(k_{u} \bar{y}\right) \cos \left(k_{u} z\right)
$$


and

$$
y_{w} \approx \frac{K_{0}}{\gamma k_{u}} \frac{\alpha}{k_{u}} \sinh \left(k_{u} \bar{y}\right) \sin \left(k_{u} z\right),
$$

where we recall that $K_{0}=e B_{0} /\left(m c k_{u}\right)$. We note that, unlike $x_{w}, y_{w}$ is nonzero only for $\bar{y} \neq 0$. Since we typically have $\alpha \bar{y} \ll 1$, the amplitude of the vertical oscillatory motion is much smaller than that of its horizontal counterpart. However, the former can still have an impact upon the vertical natural focusing (as will be seen below). Given the explicit expressions for $x_{w}$ and $y_{w}$ (as well as their slopes), we can average Eq. (A3) over the fast wiggle motion in order to obtain an equation for $\bar{x}$. We then introduce the fractional energy deviation $\delta$ through $\gamma=\gamma_{0}(1+\delta)$ and expand up to linear order in $\delta, \bar{x}$, and $\bar{y}$. The end result is

$$
\bar{x}^{\prime \prime}=\frac{e}{\gamma_{0} m c}\left[-\frac{\alpha K_{0} B_{0}}{2 \gamma_{0} k_{u}}(1+\alpha \bar{x}-2 \delta)+B_{0 c}\left(1+\alpha_{c} \bar{x}-\delta\right)\right] .
$$

Next, we stipulate that, in order for the dispersion $\eta$ to be a constant along the undulator, Eq. (A10) should admit as solutions trajectories of the form $\bar{x}=\eta \delta=$ const (in the absence of radiation). This, in turn, is only possible if we choose

$$
B_{0 c}=\frac{\alpha K_{0} B_{0}}{2 \gamma_{0} k_{u}}=\frac{B_{0}}{\gamma_{0}} \frac{1}{k_{u} \eta} \frac{2+K_{0}^{2}}{2 K_{0}}
$$

and

$$
\alpha_{c}=\alpha-\frac{1}{\eta}=\frac{2}{K_{0}^{2}} \frac{1}{\eta}=\frac{\alpha}{1+K_{0}^{2} / 2},
$$

where we have also made use of the TGU resonance condition [Eq. (2)]. Using Eqs. (A11) and (A12) along with Eq. (A10), we obtain the final form of the linearized equation of motion in the $x$ direction:

$$
\bar{x}^{\prime \prime}=-k_{\beta}^{2}(\bar{x}-\eta \delta),
$$

where $k_{\beta}$ is the total horizontal focusing strength, which is given by

$$
k_{\beta}^{2}=\frac{K_{0}^{2}}{2 \gamma_{0}^{2}} \frac{\alpha}{\eta}=\frac{1+K_{0}^{2} / 2}{\eta^{2} \gamma_{0}^{2}} .
$$

Moreover, an analogous averaging of Eq. (A4) over the wiggle motion yields

$$
\bar{y}^{\prime \prime}=-k_{y}^{2} \bar{y},
$$

where

$$
k_{y}^{2}=\frac{K_{0}^{2}}{2 \gamma_{0}^{2}}\left[k_{u}^{2}+\alpha^{2}+\alpha \alpha_{c}\right]=k_{n}^{2}+\frac{K_{0}^{2} \alpha \alpha_{c}}{2 \gamma_{0}^{2}} .
$$

Here, $k_{n}=K_{0} \sqrt{k_{u}^{2}+\alpha^{2}} /\left(\sqrt{2} \gamma_{0}\right)$ is the vertical natural focusing of the transverse gradient undulator while the remaining term denotes the vertical focusing due to the corrector field. The next step is to consider the equations which govern the longitudinal motion. Starting from the definition of the ponderomotive phase $\psi=k_{u} z+$ $k_{r}(z-c t)$, we have

$$
\frac{d \psi}{d z}=k_{u}+k_{r}\left(1-\beta_{z}^{-1}\right) \approx k_{u}-\frac{k_{r}}{2}\left(\frac{1}{\gamma^{2}}+x^{\prime 2}+y^{\prime 2}\right),
$$

where $\beta_{j}=v_{j} / c(j=x, y, z)$ are the normalized velocities and we have used the relativistic approximations $\beta_{z} \approx 1-$ $\left(1 / \gamma^{2}+\beta_{\perp}^{2}\right) / 2$ and $\beta_{\perp}^{2}=\beta_{x}^{2}+\beta_{y}^{2} \approx x^{\prime 2}+y^{\prime 2}$ (which are valid for $\gamma \gg 1$ and $\left.v_{z} \approx c\right)$. Recalling that $x^{\prime}=\bar{x}^{\prime}+x_{w}^{\prime}$, $y^{\prime}=\bar{y}^{\prime}+y_{w}^{\prime}$-and in view of Eqs. (A6) and (A8)-we obtain

$$
\begin{aligned}
\frac{d \psi}{d z}= & k_{u}-\frac{k_{r}}{2}\left(\bar{x}^{\prime 2}+\bar{y}^{\prime 2}+2 \bar{x}^{\prime} x_{w}^{\prime}+2 \bar{y}^{\prime} y_{w}^{\prime}\right) \\
& -\frac{k_{r}}{2 \gamma^{2}}\left\{1+K_{0}^{2}\left[(1+\alpha \bar{x})^{2}+\left(k_{u}^{2}+\alpha^{2}\right) \bar{y}^{2}\right] \cos ^{2}\left(k_{u} z\right)\right\} .
\end{aligned}
$$

Defining the new phase variable

$$
\theta=\psi+\frac{k_{r} K_{0}^{2}}{8 k_{u} \gamma_{0}^{2}} \sin \left(2 k_{u} z\right)
$$

and once again averaging over the fast wiggle motion eventually yields the relation

$$
\begin{aligned}
\frac{d \theta}{d z}= & 2 k_{u}(\delta-\bar{x} / \eta)-\frac{k_{r}}{2}\left(\bar{y}^{\prime 2}+k_{n}^{2} \bar{y}^{2}\right) \\
& -\frac{k_{r}}{2}\left\{\bar{x}^{\prime 2}+k_{\beta}^{2}\left[(\bar{x}-\eta \delta)(\bar{x}-3 \eta \delta)+\bar{x}^{2} / a_{w}^{2}\right]\right\} .
\end{aligned}
$$

In the process of deriving the above result, we have made use of the definition of $\delta$ and the FEL resonance condition $k_{u}=k_{r}\left(1+a_{w}^{2}\right) /\left(2 \gamma_{0}^{2}\right)$. As we have seen, the TGU is characterized by a natural focusing strength $k_{\beta} \sim\left(\eta \gamma_{0}\right)^{-1}$ in the horizontal (dispersive) direction. From Eqs. (2) and (A12), we also have $\alpha, \alpha_{c} \sim 1 / \eta$, which means that the terms proportional to $\alpha^{2}$ and $\alpha \alpha_{c}$ on the RHS of Eq. (A16) (which give the contributions to the vertical focusing due to the undulator and corrector gradients) are both of order $\left(\eta \gamma_{0}\right)^{-2} \sim k_{\beta}^{2}$. Here, we will assume that the horizontal focusing effect is very weak and neglect all terms $\sim k_{\beta}^{2}$. In this approximation, we have $k_{y} \approx k_{n} \approx K_{0} k_{u} /\left(\sqrt{2} \gamma_{0}\right)$, the transverse equations of motion are simply 


$$
\begin{gathered}
\bar{x}^{\prime \prime}=0, \\
\bar{y}^{\prime \prime}=-k_{n}^{2} \bar{y},
\end{gathered}
$$

and the phase equation reduces to

$$
\frac{d \theta}{d z}=2 k_{u}(\delta-\bar{x} / \eta)-\frac{k_{r}}{2}\left(\bar{x}^{\prime 2}+\bar{y}^{\prime 2}+k_{n}^{2} \bar{y}^{2}\right),
$$

which are the results quoted in the text (with the bars omitted). Finally, we briefly point out that the remaining part of the FEL pendulum equations follows directly from the energy exchange equation

$$
m c^{2} \frac{d \gamma}{d t}=-e E_{x} v_{x}
$$

which can also be written as

$$
\frac{d \delta}{d z} \approx-\frac{e E_{x}\left(\bar{x}^{\prime}+x_{w}^{\prime}\right)}{\gamma_{0} m c^{2}} .
$$

Utilizing Eqs. (4), (A6), and (A19)—along with the JacobiAnger expansion-and averaging over the wiggle motion, we obtain Eq. (6) in the main text. The details of this last derivation are essentially identical to the ones for a standard FEL [12] and will not be repeated here.
[1] W. Ackermann et al., Nat. Photonics 1, 336 (2007).

[2] P. Emma et al., Nat. Photonics 4, 641 (2010).

[3] T. Ishikawa et al., Nat. Photonics 6, 540 (2012).

[4] E. Allaria et al., Nat. Photonics 6, 699 (2012).

[5] E. Esarey, C. B. Schroeder, and W. P. Leemans, Rev. Mod. Phys. 81, 1229 (2009).

[6] Y. Cai, K. Bane, R. Hettel, Y. Nosochkov, M. H. Wang, and M. Borland, Phys. Rev. ST Accel. Beams 15, 054002 (2012).

[7] Y. Ding, P. Baxevanis, Y. Cai, Z. Huang, and R. Ruth, in The 4th International Particle Accelerator Conference, Shanghai, 2013.

[8] A. R. Maier, A. Meseck, S. Reiche, C. B. Schroeder, T. Seggebrock, and F. Gruner, Phys. Rev. X 2, 031019 (2012).

[9] T. Smith, J. M. J. Madey, L. R. Elias, and D. A. G. Deacon, J. Appl. Phys. 50, 4580 (1979).

[10] N. Kroll, P. Morton, M. Rosenbluth, J. Eckstein, and J. Madey, IEEE J. Quantum Electron. 17, 1496 (1981).

[11] Z. Huang, Y. Ding, and C. B. Schroeder, Phys. Rev. Lett. 109, 204801 (2012).

[12] Z. Huang and K.-J. Kim, Phys. Rev. ST Accel. Beams 10, 034801 (2007).

[13] P. Baxevanis, R. Ruth, and Z. Huang, Phys. Rev. ST Accel. Beams 16, 010705 (2013).

[14] R. Bonifacio, C. Pellegrini, and L. Narducci, Opt. Commun. 50, 373 (1984).

[15] M. Xie, Nucl. Instrum. Methods Phys. Res., Sect. A 507, 450 (2003).

[16] S. Reiche, Nucl. Instrum. Methods Phys. Res., Sect. A 429, 243 (1999). 